\title{
Service Outage based Power and Rate Allocation
}

\author{
Jianghong Luo, Lang Lin, Roy Yates, and Predrag Spasojević \\ Wireless Information Network Laboratory (WINLAB) \\ Rutgers University, 73 Brett Road, Piscataway, NJ 08854-8060 \\ e-mail: \{hongluo, llin, ryates, spasojev\}@winlab.rutgers.edu
}

\begin{abstract}
This paper explores a spectrally efficient, variable rate transmission scheme under a service outage constraint for a block fading channel model. The service outage constraint is motivated by real time variable rate applications. In a service outage, the transmission code rate is below a given basic rate and may in fact be zero. Unlike an information outage in the capacity versus outage problem, asymptotically error free communication is maintained during a service outage. We solve the problem of maximizing the time average transmission code rate subject to the probability of service outage being sufficiently small. When the problem is feasible, the optimum power policy is shown to be a combination of water filling and channel inversion allocations. The optimum power allocation for delay limited capacity, capacity versus outage, and ergodic capacity are special cases of this optimum scheme. Numerical results for the Rayleigh fading channel are given.
\end{abstract}

\section{INTRODUCTION}

Wireless communication channels vary with time due to mobility of users and changes in the environment. For a time varying channel, dynamic allocation of resources such as power, rate, or bandwidth can result in a better performance than fixed allocation strategies. Generally, the resource allocation problem requires finding the best allocation scheme given an optimization metric. The optimization metric is specified according to the quality of service (QoS) requirements of an application.

In order to differentiate real-time from non realtime applications, three capacity measures have been defined in the literature: ergodic capacity [1], delay limited capacity [2], and capacity versus outage probability $[3,4]$. A comprehensive survey of these concepts can be found in [5]. The concepts of delay limited capacity and the capacity versus outage probability have been developed in [2] and [3, 4], respectively, for applications with strict delay constraints. Although these approaches use a fixed rate transmission scheme, this may not be essential for some real time applications, including video, audio, web surfing, and games. Motivated by these applica- tions, this paper examines variable rate transmission schemes for real time applications.

For real time applications, a communication session is usually broken into frames and a certain amount of information must be transmitted within each frame. One approach to meet a real time requirement is to ensure that the transmission code rate over a frame meets a lower bound $r_{o}$, which we call the basic service rate. This basic service rate is specified by an application. We also notice that for many applications, the service quality is still deemed acceptable when the transmission code rate is occasionally lower than $r_{o}$. Based on these considerations, we propose the concept of service outage. The service is said to be in an outage when the transmission code rate over a frame is smaller than $r_{o}$. The service quality is acceptable as long as the service outage probability is less than $\epsilon$, a parameter indicating the outage tolerance of the application. Therefore, parameters $\left(r_{\mathrm{o}}, \epsilon\right)$ characterize the quality of service (QOS) requirements for a real time variable rate service.

We would like to emphasize that a service outage is fundamentally different from an information outage defined in [4]. In [4], the transmission code rate is fixed while the instantaneous mutual information fluctuates with the fading process. The instantaneous mutual information specifies the maximum reliable transmission code rate for each channel state. When the transmission code rate is greater than the instantaneous mutual information, decoding errors occur and this event is termed information outage. By comparison, in this work, the transmission code rate varies with the fading process and is always equal to the instantaneous mutual information, ensuring asymptotically error-free communication at all times. However, when the transmission code rate is less than the basic service rate, the rate requirement of the application is not satisfied and this event is termed service outage.

Since the transmission code rate varies from frame to frame, the spectral efficiency of this approach is determined by the average rate. Therefore, in this paper, the allocation problem is defined as follows: given the average power $P_{\text {av }}$, the basic service rate $r_{\mathrm{o}}$, and the maximum allowable outage probability $\epsilon$, find the optimum power and rate allocation policy to maximize the average rate.

The remainder of this paper is organized as fol- 
lows. In Section II, the system model and the optimization problem are presented. In Section III, the optimum power allocation is derived. The properties of the optimum allocation are discussed in Section IV and numerical results for the Rayleigh fading channel are given in Section V. Conclusions are drawn in Section VI.

\section{The Allocation Problem}

In this work, we employ the block flat fading Gaussian channel (BF-AWGN) model [3]. In the BFAWGN channel, a block of $N$ symbols experiences the same channel state, which is constant over the whole block, but may vary from block to block. Note that the value of $N$ is related to the product of the coherence time and the coherence bandwidth of the wireless channel. We make the following assumptions:

- The channel state information is known perfectly at both transmitter and receiver.

Within each block we have the time-invariant Gaussian channel

$$
y=\sqrt{h} x+n .
$$

Here $x$ is the channel input, $y$ is the channel output, $n$ is white Gaussian noise with variance $\sigma^{2}$, and $h$ is the channel state. Since the channel state information is known at the transmitter, an adaptive transmission scheme can be employed in the system. Let $p(h)$ denote the power allocation for a channel state $h$ and $R[h p(h)]$ be the capacity of a Gaussian channel with received power $h p(h)$, where

$$
R[P]=\frac{1}{2} \log _{2}\left(1+\frac{P}{\sigma^{2}}\right) .
$$

- One adaptive transmission frame spans one fading block and the block size $N$ is sufficiently large for reliable communication.

This assumption is reasonable as long as the product of the coherence bandwidth and the block duration is large enough. Each codeword spans only one block, and, consequently, the decoding delay is fixed and independent of the correlation structure of the fading process. As a real time requirement, we allow for the transmission code rate per frame to be only occasionally (with some probability less than or equal to $\epsilon$ ) below an application specified rate $r_{\mathrm{o}}$

- The fading process is ergodic.

Under this assumption, the time average rate is equal to the expected rate.

Let $f(h)$ denote the probability density function of the channel state $h$ and $F(h)$ denote the corresponding cumulative distribution function. Here, we only consider the case where $h$ is a continuous random variable. ${ }^{1}$ The assigned code rate for a channel state $h$ is always equal to the capacity of the Gaussian channel $R[h p(h)]$ with received power $h p(h)$. Thus, the resource allocation problem requires finding only the optimum power allocation $p^{*}(h)$. Given the average power $P_{\mathrm{av}}$, the basic service rate $r_{\mathrm{o}}$, and the allowable service outage probability $\epsilon$, we wish to maximize the expected code rate, as follows:

$$
\begin{aligned}
& R^{*}=\max _{p(h)} E_{h}\{R[h p(h)]\} \\
& \text { subject to: } \quad E_{h}\{p(h)\} \leq P_{\mathrm{av}} \\
& p(h) \geq 0 \\
& \operatorname{Pr}\left\{R[h p(h)]<r_{\mathrm{o}}\right\} \leq \epsilon .
\end{aligned}
$$

The service outage constraint (3c) reflects the real time requirement of an application. In the absence of the constraint (3c), $R^{*}$ would be the ergodic capacity for the fading channel, and the well known water filling allocation [6, 7] would be the corresponding optimum power assignment.

\section{Optimum Power And Rate Allocation}

In this section, we derive an optimum power allocation $p^{*}(h)$ for problem (3). The difficulty in deriving $p^{*}(h)$ is primarily due to the probabilistic character of the constraint (3c). Here, we show how an optimum power allocation can be derived based on a problem analogous to (3) with a deterministic constraint on the assigned rate. Given a basic service rate $r_{\mathrm{o}}$, this new constraint is based on the concept of a service set defined in the following.

Definition 1 For a power policy $p(h)$, the service set is $\mathcal{H}_{\mathrm{s}}(p(h))=\left\{h \mid R[h p(h)] \geq r_{\mathrm{o}}\right\}$, and its complement is the outage set $\mathcal{H}_{\mathrm{o}}(p(h))=\left\{h \mid R[h p(h)]<r_{\mathrm{o}}\right\}$.

Our approach will be to show that there is an optimum solution to problem (3) with a particular service set. This approach will require first solving the following subproblem in which we require that the service set contains an arbitrary set of channel states $\mathcal{H}_{a}$ :

$$
\begin{aligned}
R^{*}\left(\mathcal{H}_{a}\right)=\max _{p(h)} E_{h}\{R[h p(h)]\} \\
\text { subject to: } E_{h}\{p(h)\} \leq P_{\mathrm{av}} \\
p(h) \geq 0 \\
R[h p(h)] \geq r_{\mathrm{o}} \quad h \in \mathcal{H}_{a} .
\end{aligned}
$$

Clearly, constraint (4c) implies that $\mathcal{H}_{a}$ is a subset of the service set of any feasible policy for problem (4). Let $p^{*}\left(h, \mathcal{H}_{a}\right)$ denote the optimum solution to problem (4). Therefore, $p^{*}\left(h, \mathcal{H}_{a}\right)$ achieves the highest average rate among all the schemes whose service set contains $\mathcal{H}_{a}$.

\footnotetext{
${ }^{1}$ When $h$ is a discrete random variable, optimum power policies are typically probabilistic.
} 
Problem (4) does not necessarily have a solution given $\left(P_{a v}, r_{\mathrm{o}}, \mathcal{H}_{a}\right)$. Constraint (4c) implies that a feasible allocation $p(h)$ must satisfy

$$
p(h) \geq \frac{\sigma^{2}\left(2^{2 r_{\mathrm{o}}}-1\right)}{h} \quad h \in \mathcal{H}_{a} .
$$

This implies that the minimum average power needed to meet the constraint $(4 \mathrm{c})$, given $\left(r_{\mathrm{o}}, \mathcal{H}_{a}\right)$, is

$$
P_{\min }\left(r_{\mathrm{o}}, \mathcal{H}_{a}\right)=\int_{\mathcal{H}_{a}} \frac{\sigma^{2}\left(2^{2 r_{\mathrm{o}}}-1\right)}{h} f(h) d h .
$$

Consequently, problem (4) has a solution only if $P_{\mathrm{av}} \geq P_{\min }\left(r_{\mathrm{o}}, \mathcal{H}_{a}\right)$. When $P_{\mathrm{av}}=P_{\min }\left(r_{\mathrm{o}}, \mathcal{H}_{a}\right)$ the corresponding power allocation is

$$
p^{*}\left(h, \mathcal{H}_{a}\right)=\left\{\begin{array}{ll}
\frac{\sigma^{2}\left(2^{2 r_{\mathrm{o}}}-1\right)}{h} & h \in \mathcal{H}_{a} \\
0 & \text { otherwise }
\end{array} .\right.
$$

When $P_{\mathrm{av}}>P_{\min }\left(r_{\mathrm{o}}, \mathcal{H}_{a}\right)$ the corresponding power allocation is given by the following theorem. We use the notation $(x)^{+}=\max (x, 0)$.

Theorem 1 When $P_{\mathrm{av}}>P_{\min }\left(r_{\mathrm{o}}, \mathcal{H}_{a}\right)$ the optimum solution for problem (4) requires that:

$$
p^{*}\left(h, \mathcal{H}_{a}\right)=\left\{\begin{array}{ll}
\frac{\sigma^{2}\left(2^{2 r_{\mathrm{o}}}-1\right)}{h} & h \in \mathcal{H}_{a}^{*} \\
\sigma^{2}\left(\frac{1}{h_{0}}-\frac{1}{h}\right)^{+} & \text {otherwise }
\end{array},\right.
$$

where

$$
\mathcal{H}_{a}^{*}=\mathcal{H}_{a} \cap\left\{h \leq h_{0} 2^{2 r_{\mathrm{o}}}\right\}
$$

and $h_{0}$ is the solution of $E_{h}\left\{p^{*}\left(h, \mathcal{H}_{a}\right)\right\}=P_{\mathrm{av}}$.

Note that when $P_{\mathrm{av}}=P_{\min }\left(r_{\mathrm{o}}, \mathcal{H}_{a}\right)$, the resulting power allocation (7) can be viewed as a limiting case of expression (8) as $h_{0} \rightarrow \infty$. The power allocation $p^{*}\left(h, \mathcal{H}_{a}\right)$ is a combination of channel inversion and water filling allocations. To obtain a high average rate, we would like to allocate power in the form of the water filling allocation, while to meet the service constraint (4c), we must allocate power no less than the channel inversion allocation within the set $\mathcal{H}_{a}$. The solution $p^{*}\left(h, \mathcal{H}_{a}\right)$ balances these two factors.

Given the distribution $F(h)$ on channel states, we define $h_{\epsilon}$ such that $F\left(h_{\epsilon}\right)=\epsilon$. Note that $h_{\epsilon}$ is well defined if $h$ is a continuous random variable. The threshold $h_{\epsilon}$ partitions the channel space into a set $\mathcal{H}_{\epsilon}=\left\{h \geq h_{\epsilon}\right\}$ of good channels and the complementary set $\overline{\mathcal{H}}_{\epsilon}=\left\{h<h_{\epsilon}\right\}$ of bad channels. The following theorem indicates that the optimum scheme ought to meet the service constraint on the set of good channel states.

Theorem 2 Problem (3) has an optimum solution $p^{*}(h)$ such that $\mathcal{H}_{\epsilon} \subseteq \mathcal{H}_{\mathrm{s}}\left(p^{*}(h)\right)$.
From Theorem 2 it is easy to show that problem (3) is equivalent to problem (4) with $\mathcal{H}_{a}=\mathcal{H}_{\epsilon}$. Thus, an optimum solution is $p^{*}(h)=p^{*}\left(h, \mathcal{H}_{\epsilon}\right)$ and the following conclusions apply to problem (3).

- Problem (3) is feasible if

$$
P_{\mathrm{av}} \geq P_{\min }\left(r_{\mathrm{o}}, \mathcal{H}_{\epsilon}\right)
$$

where

$$
P_{\min }\left(r_{\mathrm{o}}, \mathcal{H}_{\epsilon}\right)=\int_{h_{\epsilon}}^{\infty} \frac{\sigma^{2}\left(2^{2 r_{\mathrm{o}}}-1\right)}{h} f(h) d h .
$$

- When $P_{\mathrm{av}}=P_{\min }\left(r_{\mathrm{o}}, \mathcal{H}_{\epsilon}\right)$ we have

$$
p^{*}(h)=\left\{\begin{array}{ll}
\frac{\sigma^{2}\left(2^{2 r_{\mathrm{o}}}-1\right)}{h} & h \geq h_{\epsilon} \\
0 & h<h_{\epsilon}
\end{array} .\right.
$$

- When $P_{\mathrm{av}}>P_{\min }\left(r_{\mathrm{o}}, \mathcal{H}_{\epsilon}\right)$, we can apply Theorem 1 with $\mathcal{H}_{a}=\mathcal{H}_{\epsilon}$ yielding

$$
p^{*}(h)=\left\{\begin{array}{ll}
\frac{\sigma^{2}\left(2^{2 r_{\mathrm{o}}}-1\right)}{h} & h \in \mathcal{H}^{*} \\
\sigma^{2}\left(\frac{1}{h_{0}^{*}}-\frac{1}{h}\right)^{+} & \text {otherwise }
\end{array},\right.
$$

where

$$
\mathcal{H}^{*}=\left\{h \geq h_{\epsilon}\right\} \cap\left\{h<h_{0}^{*} 2^{2 r_{\circ}}\right\},
$$

and $h_{0}^{*}$ is the solution of $E_{h}\left\{p^{*}(h)\right\}=P_{\mathrm{av}}$. When $h_{0}^{*} \rightarrow \infty$, this power allocation will reduce to allocation (12).

\section{Properties of The optimum ALLOCATION}

The optimum power allocation scheme (13) includes a combination of channel inversion and water filling. Depending on the value of $\left(P_{\mathrm{av}}, r_{\mathrm{o}}, \epsilon\right)$ the optimum solution belongs to one of the following possible types:

I When $P_{\mathrm{av}}=P_{\min }\left(r_{\mathrm{o}}, \mathcal{H}_{\epsilon}\right), p^{*}(h)$ includes no transmission for $h<h_{\epsilon}$ and channel inversion for $h \geq h_{\epsilon}$.

II When $P_{\text {av }}>P_{\min }\left(r_{\mathrm{o}}, \mathcal{H}_{\epsilon}\right)$ but $h_{\epsilon} \leq h_{0}^{*}, p^{*}(h)$ includes no transmission for $h<h_{\epsilon}$, channel inversion for $h_{\epsilon} \leq h<h_{0}^{*} 2^{2 r_{\mathrm{o}}}$, and water filling for $h \geq h_{0}^{*} 2^{2 r_{\mathrm{o}}}$.

III When $P_{\mathrm{av}}$ is sufficiently high such that $h_{\epsilon} 2^{-2 r_{\mathrm{o}}}<h_{0}^{*}<h_{\epsilon}, p^{*}(h)$ includes no transmission for $h<h_{0}^{*}$, water filling for $h_{0}^{*} \leq h<$ $h_{\epsilon}$, channel inversion for $h_{\epsilon} \leq h<h_{0}^{*} 2^{2 r_{\mathrm{o}}}$, and water filling for $h \geq h_{0}^{*} 2^{2 r_{\mathrm{o}}}$.

IV When $P_{\mathrm{av}}$ is high enough for $h_{0}^{*} \leq h_{\epsilon} 2^{-2 r_{\mathrm{o}}}$, $p^{*}(h)$ is just the water filling allocation. 


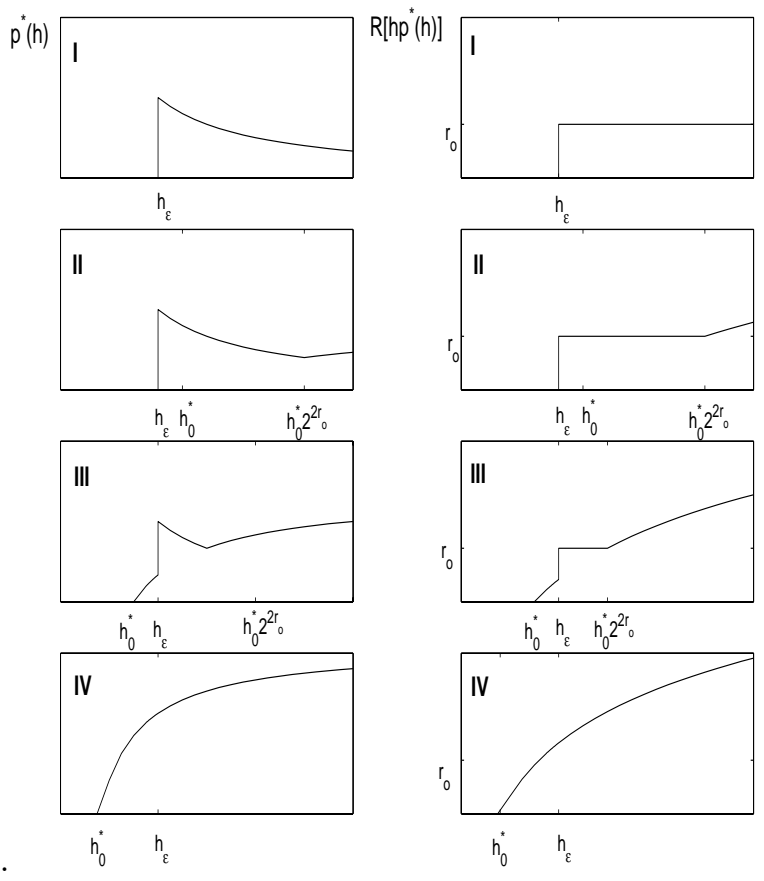

Fig. 1: For optimum solution types I-IV, power policies are given on the left and the corresponding rate allocations are on the right.

These four types of power allocation schemes as well as the corresponding rate allocations are depicted in Figure 1. For solution types I, II, and III, the optimum service set is $\mathcal{H}_{\mathrm{s}}\left(p^{*}(h)\right)=\mathcal{H}_{\epsilon}$ and the resulting outage probability is $\epsilon$, while for the type IV solution $\mathcal{H}_{\epsilon} \subseteq \mathcal{H}_{\mathrm{s}}\left(p^{*}(h)\right)$ and the resulting outage probability is less than $\epsilon$. Type I solution is the onoff channel inversion allocation. In this case, we have just enough average power to satisfy the service outage constraint. When we have extra power beyond $P_{\min }\left(r_{\mathrm{o}}, \mathcal{H}_{\epsilon}\right)$, we can allocate the power in a more efficient way to obtain a higher average rate and, at the same time, to meet the service outage constraint. When $P_{\text {av }}$ is sufficiently high for the water filling allocation to satisfy the service outage constraint, then it must also be the optimum solution for problem (3). Thus, for a given pair $\left(r_{\mathrm{o}}, \epsilon\right)$, the optimum power allocation scheme gradually changes from the on-off channel inversion allocation to the water filling allocation as $P_{\text {av }}$ increases.

Now we examine the connection of the service outage problem with the capacity versus outage problem in [4]. In [4], the maximum $\epsilon$-achievable rate [8] for the general $M$-block fading channel is derived for a given average power $P_{\mathrm{av}}$ and an outage probability $\epsilon$. As a special case, the $\epsilon$-achievable rate $C_{\epsilon}\left(P_{\mathrm{av}}\right)$ for the one block fading channel is

$C_{\epsilon}\left(P_{\mathrm{av}}\right)=\sup _{E(p(h)) \leq P_{\mathrm{av}}} \sup \{R: \operatorname{Pr}\{R(h p(h))<R\} \leq \epsilon\}$
In this case, the corresponding optimum power allocation is the on-off channel inversion and the resulting $C_{\epsilon}\left(P_{\mathrm{av}}\right)$ is

$$
C_{\epsilon}\left(P_{\mathrm{av}}\right)=\frac{1}{2} \log _{2}\left(1+\frac{P_{\mathrm{av}}}{\int_{h_{\epsilon}}^{\infty}\left(\sigma^{2} / h\right) f(h) d h}\right) .
$$

If we replace the rate $R$ in expression (15) with the basic service rate $r_{\mathrm{o}}$, then $C_{\epsilon}\left(P_{\mathrm{av}}\right)$ specifies the maximum supportable basic service rate for a given $P_{\text {av }}$ and $\epsilon$ in problem (3). Therefore, problem (3) has a solution iff $r_{\mathrm{o}} \leq C_{\epsilon}\left(P_{\mathrm{av}}\right)$, or equivalently $P_{\mathrm{av}} \geq P_{\min }\left(r_{\mathrm{o}}, \epsilon\right)$ given by the feasibility condition (10). Thus, the $\epsilon$-capacity for the capacity versus outage problem provides the feasibility condition for the service outage problem and identifies the optimum power allocation when $r_{\mathrm{o}}=C_{\epsilon}\left(P_{\mathrm{av}}\right)$. This work, in the general case, determines an optimum power allocation for applications whose basic service rate requirement $r_{\mathrm{o}}$ is less than or equal to $C_{\epsilon}\left(P_{\mathrm{av}}\right)$. Furthermore, we can see that the resulting average rate $R^{*}$ satisfies $R^{*} \geq C_{\epsilon}\left(P_{\mathrm{av}}\right)(1-\epsilon)$.

We also observe that type I solution has the same on-off channel inversion power allocation form as that for the capacity versus outage problem in the one block fading channel [4]. It degenerates to the pure channel inversion scheme when $\epsilon=0$ as in the case of delay limited capacity [2]. Furthermore, as we said before, type IV solution has the same water filling power allocation form as that for ergodic capacity [1]. Thus, the optimum power allocations for minimum outage probability, delay-limited capacity, and ergodic capacity are special cases of the optimum power allocation (13) for certain parameters $\left(P_{\mathrm{av}}, r_{\mathrm{o}}, \epsilon\right)$.

\section{Numerical Results}

In this section, we apply the service outage based optimum policy to the Rayleigh fading channel. For Rayleigh fading with normalized mean, we have

$$
f(h)=\left\{\begin{array}{ll}
e^{-h} & h \geq 0 \\
0 & \text { otherwise }
\end{array} .\right.
$$

In addition, the noise variance is also normalized as $\sigma^{2}=1$.

First, the average rate versus the preset allowable service outage probability is plotted in Figure 2 with fixed $r_{\mathrm{o}}=1 \mathrm{bits} / \mathrm{symbol}$ and $P_{\mathrm{av}}=14 \mathrm{~dB}$. In this case, the feasibility condition (10) requires that parameter $\epsilon \geq \epsilon_{\min }=1.3 \cdot 10^{-4}$. The ergodic capacity is also given in Figure 2 for comparison and is 2.03 bits $/$ symbol for $P_{\mathrm{av}}=14 \mathrm{~dB}$. It can be seen that the average rate increases rapidly with the service outage probability for fixed $r_{\mathrm{o}}$ and $P_{\mathrm{av}}$. This phenomenon is easily understood. When $\epsilon=\epsilon_{\min }$, the power allocation scheme is the on-off channel inversion scheme as in [4], which is very inefficient with respect to the average rate. When $\epsilon>\epsilon_{\min }$, 


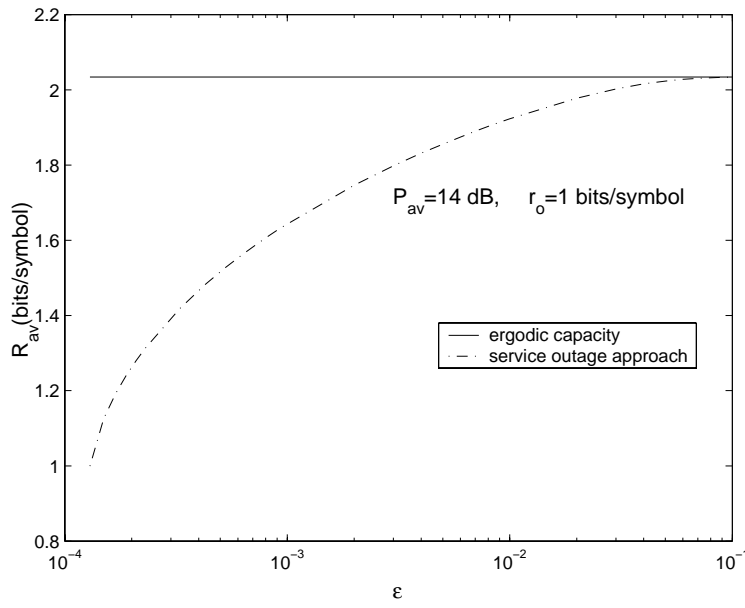

Fig. 2: Average rate versus service outage probability for a Rayleigh fading channel.
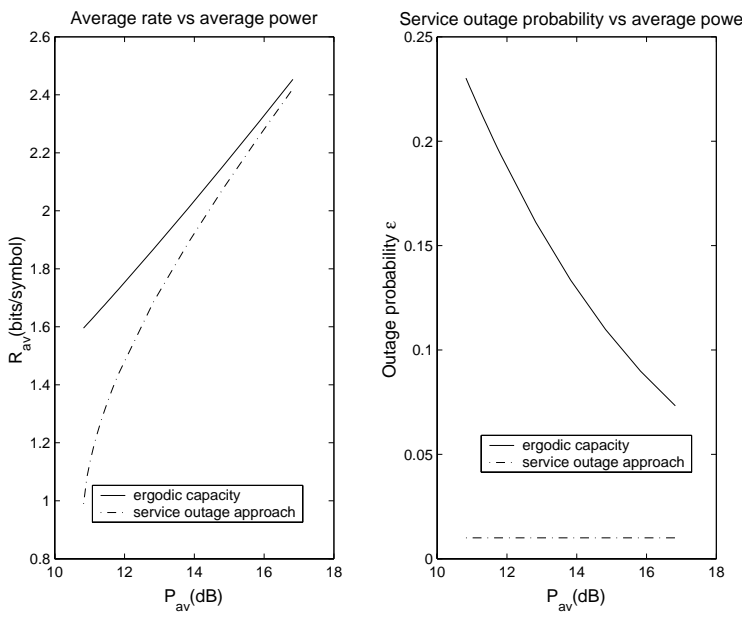

Fig. 3: Service outage approach versus water filling allocation scheme for fixed $r_{\mathrm{o}}=1 \mathrm{bits} / \mathrm{symbol}$ and $\epsilon=0.01$.

the power allocation scheme contains the water filling component, which is the most efficient allocation component for a high average rate. Therefore, one can get a much higher average rate if the application can tolerate somewhat higher service outage probability than $\epsilon_{\min }$. In this example, the average rate will achieve the ergodic capacity if the application can tolerate a service outage probability around 0.13 .

In Figure 3, the service outage approach for fixed $r_{\mathrm{o}}=1 \mathrm{bits} / \mathrm{symbol}$ and preset $\epsilon=0.01$ is compared to the pure water filling allocation. The pure water filling allocation is the optimum power allocation for the ergodic capacity. Therefore, it achieves a higher average rate than the service outage approach, but suffers from a larger service outage probability. When the average power $P_{\mathrm{av}} \geq 15 \mathrm{~dB}$ in this example, the service outage approach achieves an average

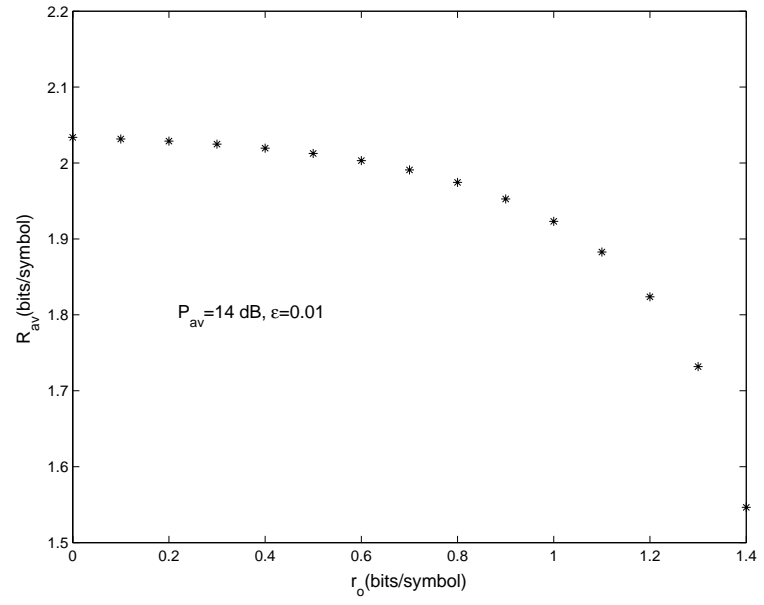

Fig. 4: Average rate versus basic service rate for a Rayleigh fading channel.

rate very close to the ergodic capacity, and, at the same time, has a much lower outage probability. Furthermore, the service outage approach meets the service outage constraint without sacrificing too much spectral efficiency.

The average rate versus the basic service rate is plotted in Figure 4 with fixed $P_{\mathrm{av}}=14 \mathrm{~dB}$ and $\epsilon=0.01$. In this case, the feasible range of the basic service rate $r_{\mathrm{o}}$ is between 0 and $1.4 \mathrm{bits} / \mathrm{symbol}$. When $r_{\mathrm{o}}=0$, the ergodic capacity is obtained. The average rate decreases with the basic service rate $r_{\mathrm{o}}$.

\section{Conclusion}

In this paper, we have identified the optimum power and rate allocation scheme in the fading channel under a service outage constraint. A service outage occurs when the transmission code rate is lower than a specified basic service rate. The service quality is deemed acceptable as long as the service outage probability is sufficiently small. Under the assumptions that one frame experiences a single channel state and that the channel state is known perfectly at the transmitter, we verify that the resulting optimum power allocation is a combination of channel inversion and water filling when the parameters $\left(P_{\mathrm{av}}, r_{\mathrm{o}}, \epsilon\right)$ are feasible. The water filling allocation maximizes the average rate without the service outage constraint, while the channel inversion allocation provides sufficient power for meeting the service outage constraint. Our solution balances these two factors. When the average power $P_{\mathrm{av}}$ is just sufficient to meet the service outage condition, the optimum power allocation has the same form as the allocation for the capacity versus outage problem in [4]. On the other hand, when $P_{\text {av }}$ is large enough, the optimum power allocation is the water filling allocation and the resulting average rate is equal to the ergodic capacity. We also 
show that the feasible parameter set $\left(P_{\mathrm{av}}, r_{\mathrm{o}}, \epsilon\right)$ can be obtained based on the capacity versus outage con-

cept [4]. In fact, the maximum supportable $r_{o}$ for a given $\epsilon$ and $P_{\text {av }}$ equals the $\epsilon$-capacity.

\section{REFERENCES}

[1] A. J. Goldsmith and P. P. Varaiya, "Capacity of fading channels with channel side information," IEEE Transactions on Information Theory, vol. 43, no. 6, pp. pp. 1986-1992, Nov. 1997.

[2] S. V. Hanly and D. N. C. Tse, "Multiaccess fading channels: part II: Delay-limited capacities," IEEE Transactions on Information Theory, vol. 44, no. 7, pp. 2816-2831, Nov. 1997.

[3] L. H. Ozarow, S. Shamai, and A. D. Wyner, "Information theoretic considerations for cellular mobile radio," IEEE Transactions on Vehicular Technology, vol. 43, no. 2, pp. 359-378, May 1994.

[4] G. Caire, G. Taricco, and E. Biglieri, "Optimum power control over fading channels," IEEE Transactions on Information Theory, vol. 45, no. 5, pp. 1468-1489, July 1999.

[5] E. Biglieri, J. Proakis, and S. Shamai, "Fading channels: Information-theoretic and communications aspects," IEEE Transactions on Information Theory, vol. 44, no. 6, pp. 2619-2692, Oct. 1998.

[6] T. Cover and J. Thomas, Elements of Information Theory, John Wiley Sons, Inc., 1991.

[7] R. Gallager, Information Theory and Reliable Communication, John Wiley and Sons, 1968.

[8] S. Verdu, "A general formula for channel capacity," IEEE Transactions on Information Theory, vol. 40, no. 4, pp. 1147-1157, July 1994. 